\title{
Magnetization reversal and magnetic interactions in patterned spin valve structures
}

\author{
Frank Brüssing ${ }^{1}$, Gregor Nowak ${ }^{1}$, Alexandra Schumann ${ }^{1}$, \\ Stefan Buschhorn ${ }^{1}$, Hartmut Zabel ${ }^{1}$ and Katharina Theis-Bröhl ${ }^{1,2}$ \\ ${ }^{1}$ Department of Physics, Ruhr-University Bochum, D-44780 Bochum, Germany \\ ${ }^{2}$ University of Applied Sciences Bremerhaven, An der Karlstadt 8, D-27568 Bremerhaven, Germany
}

Received 14 April 2009, in final form 1 July 2009

Published 31 July 2009

Online at stacks.iop.org/JPhysD/42/165001

\begin{abstract}
We have investigated the magnetization reversal and the magnetic anisotropy of epitaxial

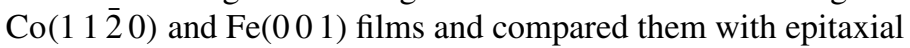

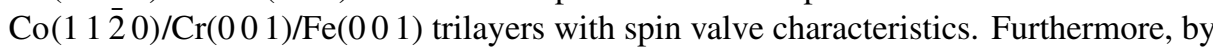
e-beam lithography we have cut the single layers and the trilayers into the shape of wires to study the effect of the shape anisotropy and the dipolar interaction between the wires on their switching characteristics. We found that patterning very much affects the magnetization reversal of the single films by introducing an additional anisotropy term. Dipolar interaction between the different wires may exist but is less obvious. However, the remagnetization process of the patterned trilayers resembles more those of the continuous single films than a combination of the Co and Fe wires. We argue that this is due to a weak but remaining interlayer exchange coupling between $\mathrm{Co}$ and $\mathrm{Fe}$, mediated by the intervening $\mathrm{Cr}$ layer.
\end{abstract}

(Some figures in this article are in colour only in the electronic version)

\section{Introduction}

The giant magneto-resistance (GMR) effect, discovered by Peter Grünberg and Albert Fert [1, 2], required for its operation the long range and oscillatory interlayer exchange coupling (IEC) between two ferromagnetic layers (F), mediated by a non-magnetic normal metal $(\mathrm{N})$ spacer layer. For specific and well-defined spacer layer thicknesses the adjacent $\mathrm{F}$ layers are coupled antiferromagnetically in remanence. In an external magnetic field the layer magnetization of both layers switches to the parallel orientation, thereby lowering the magnetoresistance. Soon after, it was noticed that IEC is not intrinsic to the GMR effect [3,4]. If one layer magnetization direction is fixed in space and the other one is essentially free to rotate via an external field, the thickness of $\mathrm{N}$ can be increased from about $1 \mathrm{~nm}$ to several nanometres, the upper limit is only restricted by the spin diffusion length, which can reach several hundred nanometres [5]. Usually one of the $F$ layers is pinned via exchange bias to an antiferromagnetic (AF) layer [4]. The layer sequence required for the operation of a GMR device is thus $\mathrm{F} / \mathrm{N} / \mathrm{F} / \mathrm{AF}$, which was termed a 'spin valve'. Instead of pinning one of the layers via coupling to an antiferromagnet, alternatively one may use two different ferromagnetic layers, one magnetically soft layer $\mathrm{F}_{1}$ with a coercive field $H_{\mathrm{c} 1}$ and another magnetically harder layer $\mathrm{F}_{2}$ with a coercive field $H_{\mathrm{c} 2}>H_{\mathrm{c} 1}$ [3]. The layer sequence is then $\mathrm{F}_{1} / \mathrm{N} / \mathrm{F}_{2}$. In an external field the soft layer will switch first, followed by the harder layer at higher fields. In the intermediate field range $H_{\mathrm{c} 1}<H<H_{\mathrm{c} 2}$ both layers are antiparallel oriented, as required for the GMR operation. Furthermore, the fabrication of $F_{1} / N / F_{2}$ type spin valves is easier than that of $F / N / F / A F$ spin valves, as deposition of the AF layer can be dispensed. $\mathrm{Co} / \mathrm{Cr} / \mathrm{Fe}$ trilayers with thick $\mathrm{Cr}$ spacer layers have been shown in the past to be excellent examples for a spin valve type magnetization reversal [6], and the GMR effect has been determined by Buchmeier et al [7].

For practical applications in spin valve devices the trilayers need to be patterned in the lateral direction. Lateral patterning of thin films has two effects. Depending on the shape and aspect ratio new magnetic anisotropies may be introduced. And second, depending on the distance between individual spin valves interaction and correlation effects may occur. For functional use with high packing density both effects call for attention. In previous studies 
on Co nanowires, correlation effects during switching of the wires were observed [8]. The aim of this study is to extend these investigations to laterally pattern $\mathrm{Co} / \mathrm{Cr} / \mathrm{Fe}$ trilayers and to decide whether their switching behaviour is correlated or independent. In the latter case $\mathrm{Co} / \mathrm{Cr} / \mathrm{Fe}$ spin valves could be useful for high density magnetic random access memory (MRAM) integration.

\section{Sample preparation}

The epitaxial layers were grown by molecular beam epitaxy (MBE) on epi-polished $\mathrm{MgO}\left(\begin{array}{ll}0 & 0\end{array}\right)$ substrates. The pressure during film deposition was better than $1 \times 10^{-9}$ mbar. A $30 \mathrm{~nm}$ thick $\mathrm{Cr}\left(\begin{array}{ll}0 & 0\end{array}\right)$ buffer layer grown at $450{ }^{\circ} \mathrm{C}$ and subsequently annealed at $750^{\circ} \mathrm{C}$ is necessary for a good epitaxial growth of the $\mathrm{Co} / \mathrm{Cr} / \mathrm{Fe}$ spin valve structure. $\mathrm{Cr}$ exhibits a so-called $45^{\circ}$-epitaxy on $\mathrm{MgO}\left(\begin{array}{lll}0 & 0 & 1\end{array}\right)$ with the $\left[\begin{array}{lll}1 & 0 & 0\end{array}\right]$ crystallographic axes of both materials being aligned at an angle of $45^{\circ}$ to each other [9].

All subsequent layers were grown at a reduced substrate temperature of $300^{\circ} \mathrm{C}$. This temperature was found to be an optimum between good epitaxial growth and low interfacial mixing. The epitaxy of $\mathrm{Fe}$ on the $\mathrm{Cr}$ buffer is pseudomorphic because of a lattice mismatch of only $0.6 \%$ between both bec materials. In contrast, $\mathrm{Co}$ is a hep material and grows

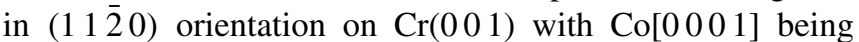
parallel to $\mathrm{Cr}[110][10,11]$. However, because of similarities between the $h c p(11 \overline{2} 0)$ and the bcc(0 01$)$ surfaces and small lattice mismatches of $0.07 \%$ parallel to $\mathrm{Co}\left[\begin{array}{llll}0 & 0 & 0 & 1\end{array}\right]$ but $6.4 \%$ along $\operatorname{Co}\left[\begin{array}{lll}1 & 1 & 2\end{array} 0\right]$, Co grows pseudomorphically on $\operatorname{Cr}\left(\begin{array}{lll}0 & 0 & 1\end{array}\right)$ by adopting its bcc structure. Subsequently, Co undergoes a continuous bcc-hcp phase transition during growth over a thickness range of 4-5 nm [10]. Because of two equivalent

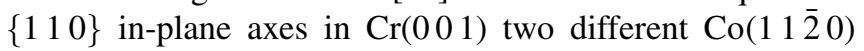
domains form with an angle of $90^{\circ}$ to each other. The thickness of the Crinterlayer between $\mathrm{Co}$ and $\mathrm{Fe}$ was chosen to be $3.1 \mathrm{~nm}$ to mediate a weak antiferromagnetic (AF) coupling as was found in [6]. The thicknesses of both magnetic layers $(4.0 \mathrm{~nm}$ for $\mathrm{Co}$ and $3.0 \mathrm{~nm}$ for $\mathrm{Fe}$ ) were chosen to be different due to their different magnetic moments and with the intention of a cancellation of the film magnetic moment in the antiparallel state of $\mathrm{Co}$ and $\mathrm{Fe}$ layer magnetization. The layers were capped with $\mathrm{Cr}$ for oxidation protection.

The quality of the samples was verified in situ by reflection high energy electron diffraction (RHEED) and ex situ by x-ray scattering. In figure 1 a representative set of RHEED pictures taken from the $\mathrm{Fe}$, the $\mathrm{Cr}$ and the $\mathrm{Co}$ surfaces are shown with streaks oriented along the $\mathrm{MgO}\left[\begin{array}{lll}1 & 0 & 0\end{array}\right]$ and the $\mathrm{MgO}\left[\begin{array}{lll}1 & 1 & 0\end{array}\right]$ orientation. Additionally, a RHEED picture taken from the Co surface with streaks parallel to the $\mathrm{Co}\left[\begin{array}{llll}1 & 1 & 0 & 1\end{array}\right]$ direction is presented. Here Co is already in the hcp phase as can be verified by the existence of streaks in these directions which is not the case in $\mathrm{Cr}$ nor in the Co bcc phase. The presence of the Co hcp phase can also be verified by additional weak reflections along the $\mathrm{MgO}\left[\begin{array}{lll}1 & 1 & 0\end{array}\right]$ orientation as compared with the equivalent $\mathrm{Cr}$ RHEED pattern.

After growth the films were patterned into the form of stripes of $2 \mu \mathrm{m}$ width and with a periodicity of $3 \mu \mathrm{m}$ by using
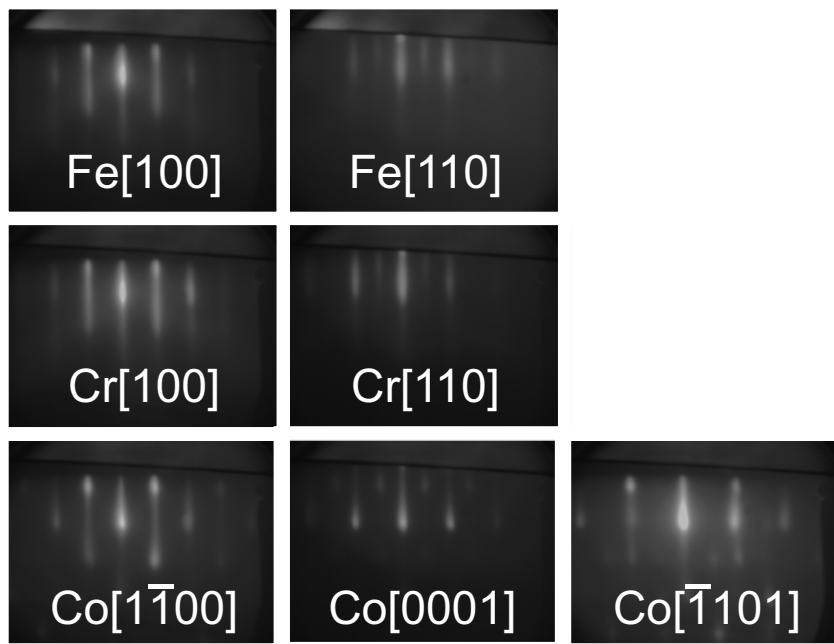

Figure 1. RHEED pictures taken from different surfaces parallel to the $\mathrm{MgO}\left[\begin{array}{lll}1 & 1 & 0\end{array}\right]$ orientation (left), to the $\mathrm{MgO}\left[\begin{array}{lll}1 & 0 & 0\end{array}\right]$ orientation (middle) and from the Co surface parallel to the Co[ $\left.\begin{array}{llll}\overline{1} & 1 & 0 & 1\end{array}\right]$ orientation (right).

electron-beam (e-beam) lithography and ion beam etching techniques. A negative resist was spin coated on the single films and the trilayer structure, exposed to the e-beam, and subsequently etched by a neutralized Ar-ion beam. The stripe orientation was chosen to be parallel to one of the easy axes. A schematic drawing of the sample design for the spin valve structures is shown in figure 2 .

\section{Experimental results}

\subsection{Structural properties}

3.1.1. Unpatterned samples. X-ray measurements were performed to verify the layer thicknesses, the quality of the interfaces and the epitaxy of the films. For these measurements an x-ray source of high brilliance is indispensable to analyse the structural properties of such a complex system. Therefore, we used the synchrotron beam line (BL9) of the DELTA synchrotron at the Technical University Dortmund with an incident energy of $11 \mathrm{keV}$. Figure 3 shows x-ray reflectivity data from a sample with a design as shown in the left panel of figure 2. Both well-defined oscillations and a linear drop in the intensity on a logarithmic scale indicate a high quality of layers and interfaces as well as very smooth interfaces. Transverse scans across the reflectivity curve are shown in the inset and feature Yoneda wings on both sides of a narrow central peak, which is indicative of some structural roughness. The double peak structure at the specular peak position can be explained by at least two different surface areas which are slightly canted against each other. This observation is often made from $\mathrm{MgO}$ substrates and may occur during polishing of large surface areas.

The out-of-plane crystalline quality of the sample is revealed by a longitudinal high angle scan as shown in figure 4 . Sharp $\mathrm{Fe}(002)$ and $\operatorname{Co}(11 \overline{2} 0)$ peaks indicate an excellent crystal quality. Similar lattice parameters of $0.2867 \mathrm{~nm}$ for $\mathrm{Fe}$ and $0.2885 \mathrm{~nm}$ for $\mathrm{Cr}$ lead to one common $\mathrm{Fe} / \mathrm{Cr}(002)$ peak 


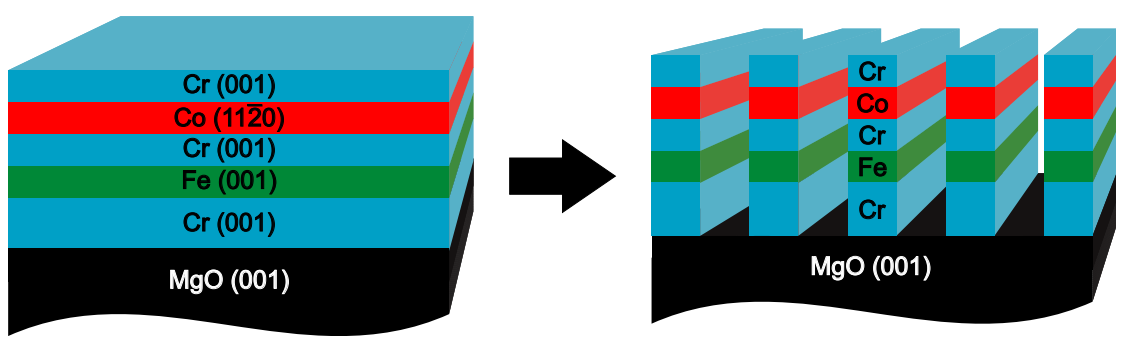

Figure 2. Schematic sample design of unpatterned and patterned samples used for this study. (Colour online.)

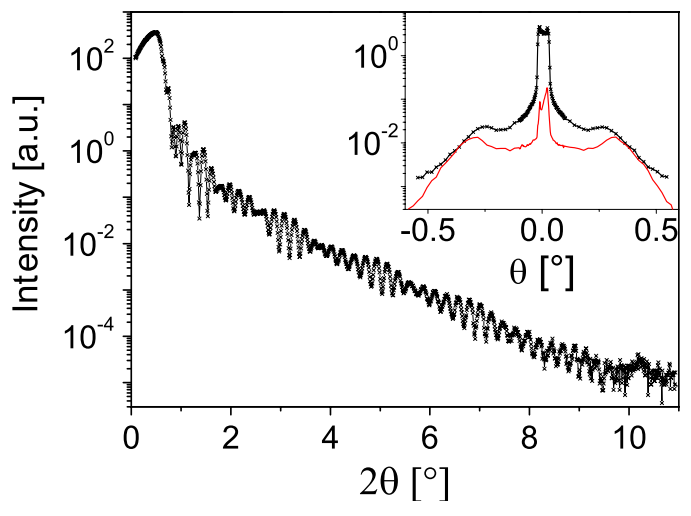

Figure 3. Specular reflectivity taken from a sample with a layer sequence as shown in figure 2 . Inset: rocking curves across the reflectivity taken at $2 \theta=1.01^{\circ}$ and $2 \theta=1.16^{\circ}$. (Colour online.)

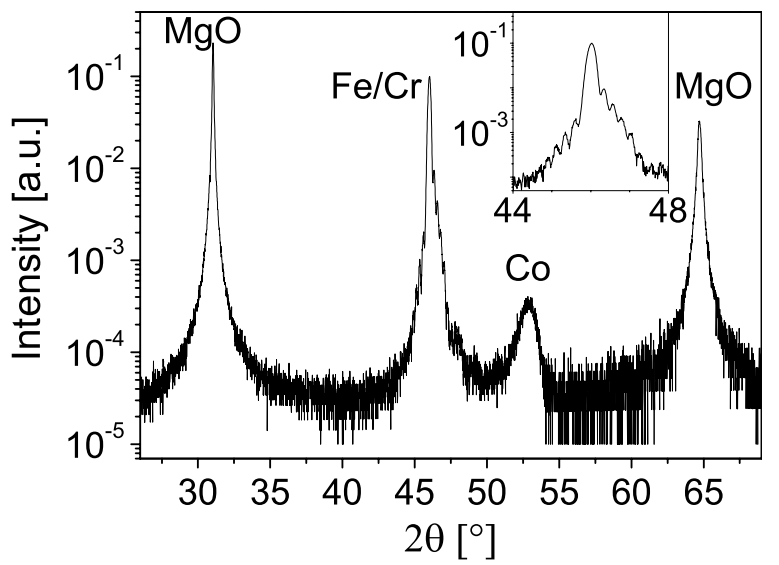

Figure 4. High angle $\mathrm{x}$-ray scan taken from a $\mathrm{Cr} / \mathrm{Co} / \mathrm{Cr} / \mathrm{Fe} / \mathrm{Cr} / \mathrm{MgO}$ sample with a layer sequence as shown in figure 2. Inset: magnification of the $\mathrm{Fe} / \mathrm{Cr}$ peak with Laue oscillations at both sides.

at $2 \theta=46.03^{\circ}$, together with Laue oscillations on either side, as shown in the inset of figure 4 . The intensity of this peak is much higher than that of the $\operatorname{Co}(11 \overline{2} 0)$ peak and is determined mainly by the thick Cr buffer layer. Furthermore, the width of the Co peak can be explained by its finite thickness.

To ensure the epitaxial quality we additionally performed in-plane Bragg scans for different sample orientations. Therefore, we used surface scattering techniques with glancing incident and exit angles. The incident angle was kept constant and equal to the critical angle for total external reflection from the surface. The intensity of the exit beam was integrated over a wide range of exit angles via a wide open detector slit. With this set-up the structural information is averaged over the entire

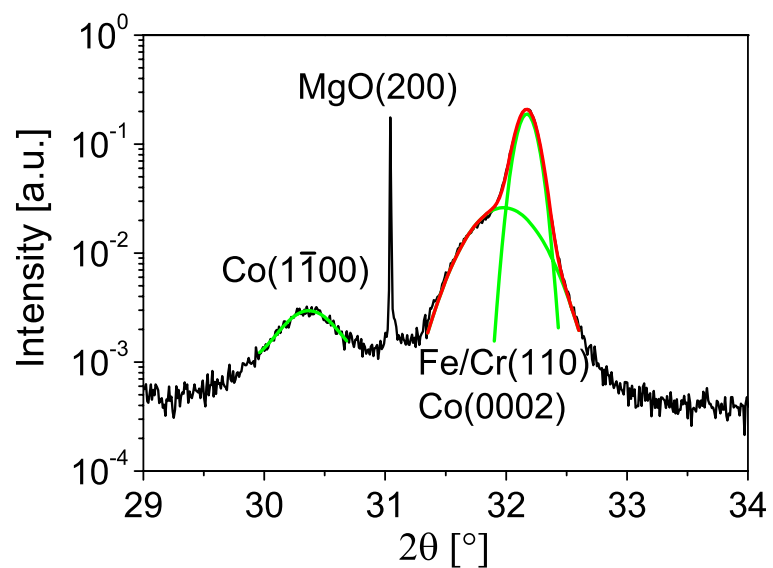

Figure 5. In-plane Bragg scan parallel to the $\mathrm{MgO}[100]$ direction. (Colour online.)

thickness. After identifying a particular in-plane reflection we performed an in-plane $\theta: 2 \theta$ radial scan across this reflection. This scan reveals the quality of the different layers by the full width at half maximum (FWHM) of their respective Bragg peaks.

One example for a measurement across the $\mathrm{MgO}(200)$ in-plane Bragg reflection is shown in figure 5. This scan includes the $\operatorname{Co}(1 \overline{1} 00)$ peak on the low angle side of the $\mathrm{MgO}(200)$ peak and a superposition of a sharp peak from $\mathrm{Fe} / \mathrm{Cr}\left(\begin{array}{lll}1 & 1 & 0\end{array}\right)$ with a FWHM of less than $1^{\circ}$ and a broader peak from $\mathrm{Co}\left(\begin{array}{lll}0 & 0 & 0\end{array}\right)$ on the high angle side of $\mathrm{MgO}(200)$. From these scans a pseudomorphic growth of $\mathrm{Fe}$ on the $\mathrm{Cr}$ buffer layer can be verified as well as a $45^{\circ}$ epitaxial relation between $\mathrm{Fe} / \mathrm{Cr}$ and $\mathrm{MgO}$.

From these measurements it is possible to calculate the coherent scattering volume for $\mathrm{Co}$ and for $\mathrm{Cr} / \mathrm{Fe}$. The coherent scattering volume can be taken as equivalent to the crystal grain size. The value for Co is $15 \mathrm{~nm} \times 15 \mathrm{~nm} \times 3 \mathrm{~nm}$. For the $\mathrm{Fe}$ and $\mathrm{Cr}$ peaks a coherent scattering volume of $40 \mathrm{~nm} \times 40 \mathrm{~nm} \times 25 \mathrm{~nm}$ can be estimated. The smaller inplane grain size of the Co film as compared with the $\mathrm{Cr} / \mathrm{Fe}$ layer may be due to the bcc-hcp phase transition in the former layer. The perpendicular coherence volume is essentially given by the film thickness.

Finally, we investigated the relative epitaxial relationship of the different layers via azimuthal scans as shown in figure 6. For these measurements we kept the absolute value of the scattering vector fixed to the particular maximum of the respective Bragg peak intensity and performed scans of the in-plane sample orientation over a range of $360^{\circ}$. We recognize 
the parallel orientation for the $\mathrm{Cr}\left\{\begin{array}{llll}1 & 1 & 0\end{array}\right\}$ and the $\operatorname{MgO}\left\{\begin{array}{lll}1 & 0 & 0\end{array}\right\}$ in-plane axes showing the $45^{\circ}$ epitaxial relation between $\mathrm{Cr}$ and $\mathrm{MgO}$ as already demonstrated in figure 5. The hcp structure of the cobalt film is clearly visible in the Co in-plane azímuthal scan, showing 8 peaks resulting from two different $\operatorname{Co}\left(\begin{array}{lll}1 & 1 & \overline{2}\end{array}\right)$ domains with an angle of $90^{\circ}$ to each other [11]. These crystallographic results allow a clear determination of the sample orientation with respect to the easy and hard magnetic axes. This, in turn, is important for the sample orientation during e-beam lithography.

3.1.2. Patterned samples. The quality of the patterned samples was investigated by scanning electron microscopy (SEM) using a secondary electron detector (SED) and an energy dispersive detector (EDX). A representative intensity

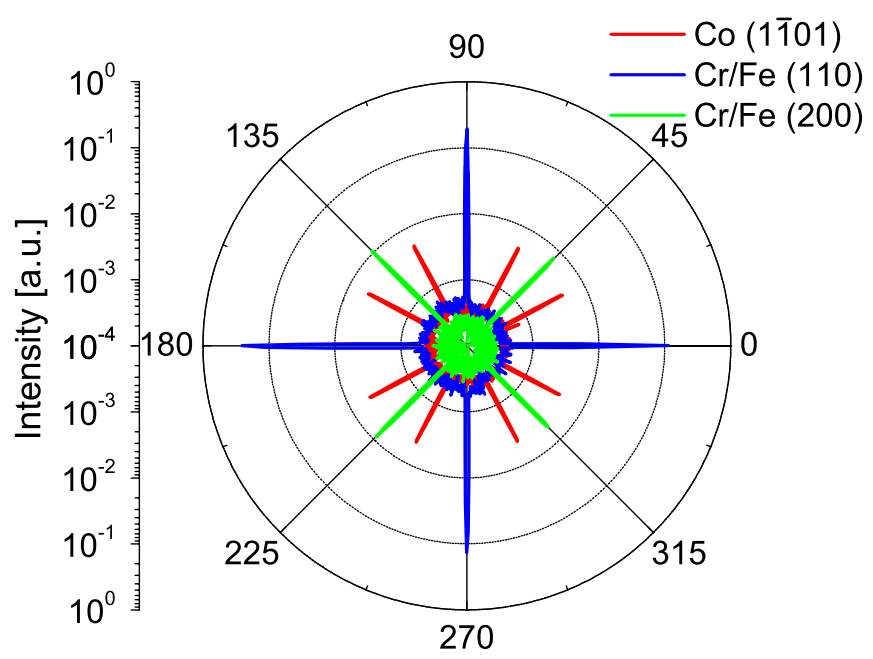

Figure 6. Results of in-plane azimuthal scans of the $\mathrm{Cr} / \mathrm{Fe}(200)$, the $\mathrm{Cr} / \mathrm{Fe}\left(\begin{array}{lll}1 & 1 & 0\end{array}\right)$ and the $\mathrm{Co}\left(\begin{array}{llll}1 & 1 & 0 & 1\end{array}\right)$ in-plane Bragg reflections. (Colour online.) map with a SED is reproduced in the top left panel of figure 7. The shape and the periodicity of the stripes can be observed with this method. However, the trenches between the stripes are not easy to confirm via this method. Therefore, we have taken EDX maps on the $\mathrm{K}_{\alpha}$ edges of the respective elements of the substrate and the trilayer stripes, giving information on the local position of the different metals in the sample. Although the resolution of this method is on the order of the width of the trenches to be investigated, the EDX maps of $\mathrm{Co}, \mathrm{Fe}$ and $\mathrm{Cr}$ confirm exactly the parameters of the sample design, including their width and separation. Vice versa, the elements of the substrate are visible in the trenches. The profile of the stripes is also confirmed by integrated intensity scans shown in figure 8 for the different elements along the lines indicated in the top left panel. From these measurements we can safely assume that the stripes are well separated and that any direct magnetic contact is rather unlikely.

For additional structural information on the patterns, atomic force microscopy (AFM) measurements were performed. In figure 9 the left panel shows an AFM image with a clear contrast with respect to the shape of the stripes. The separation of the magnetic films is clearly visible by a plot of the profile of the stripes as shown in the right image of figure 9 and displaying a height of more than $50 \mathrm{~nm}$. This is much more than the thickness of the four top layers which is only around $14 \mathrm{~nm}$. Thus the etched trenches between the stripes penetrate deep into the substrate and clearly separate the different stripes.

The structural quality of the stripe array is also expressed by Bragg reflections from the artificial lateral periodic structure. We have confirmed this by soft x-ray scattering using a resonant enhancement of the signal by tuning the $\mathrm{x}$-ray energy to the $L_{2}$ edge of $\mathrm{Fe}$. The experiments were done using the X-ray resonant magnetic scattering chamber ALICE at the BESSYII synchrotron facility. Figure 10 shows the result from a rocking curve in an angular range of $\pm 5^{\circ}$ with the detector
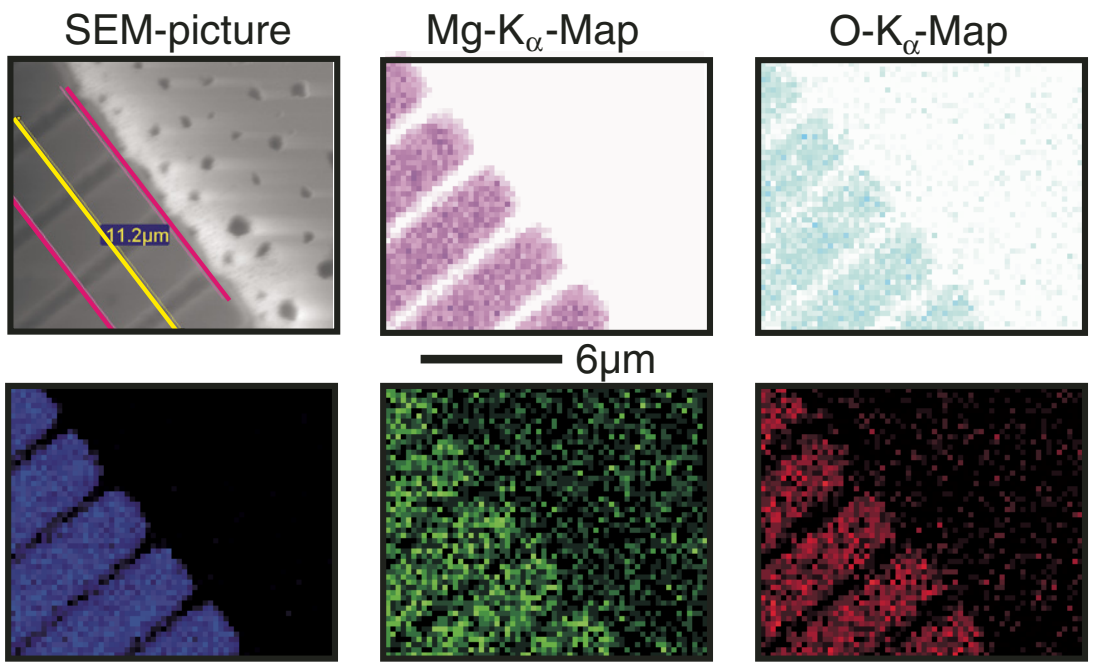

$\mathrm{Cr}-\mathrm{K}_{\alpha}-\mathrm{Map}$

$\mathrm{Fe}-\mathrm{K}_{\alpha}-\mathrm{Map}$

Co- $\mathrm{K}_{\alpha}-\mathrm{Map}$

Figure 7. SEM and energy dispersive maps of a patterned sample according to the layer sequence shown in figure 2. SEM measurement providing basic structural information, and EDX maps performed at the $\mathrm{K}_{\alpha}$ edges of the respective materials $\mathrm{O}$ (top row, middle), Mg (top row, right), $\mathrm{Cr}$ (bottom row, left), $\mathrm{Fe}$ (bottom row, middle) and $\mathrm{Co}$ (bottom row, right). The lines indicate integrated intensity scans shown in figure 8. (Colour online.) 
fixed at $10^{\circ}$. Bragg peaks can be observed up to very high orders. Furthermore, it should be noted that the intensity of every third peak is reduced due to the $2: 1$ ratio between the thickness of the Fe stripes and the distance between them.

\subsection{MOKE magnetometry}

The magnetic properties of the patterned and unpatterned structures were studied via the vector-magneto-optical Kerr effect (V-MOKE) [12]. Figure 11 shows the dependence of the remanent magnetization on the sample orientation for continuous and single $\mathrm{Fe}$ and $\mathrm{Co}$ films, and for an $\mathrm{Co} / \mathrm{Cr} / \mathrm{Fe}$ trilayer. All three samples display a fourfold anisotropy with the magnetic easy axes directed along the sample diagonal. A comparison with the $\mathrm{x}$-ray in-plane data confirms that the easy axes are aligned along the in-plane $\left\{\begin{array}{lll}1 & 0 & 0\end{array}\right\}$ and the

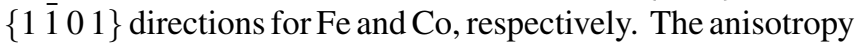
of Fe agrees with the reported anisotropy for $(100)$ oriented thin films [13]. For Co $(11 \overline{2} 0)$ oriented films, however, one would expect a strong uniaxial anisotropy with the easy axis along [0 $\left.0 \begin{array}{llll}0 & 0 & 1]\end{array}\right]$. The fact that we observe a fourfold

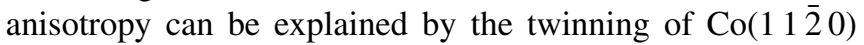
on the $\operatorname{Cr}(001)$ surface with two degenerate structural and magnetic domains [14]. The combined magnetic anisotropy of the trilayer system is qualitatively the same as for the single layers.

While the magnetic anisotropy directions of the single films and the trilayer are identical, their coercivities are hugely

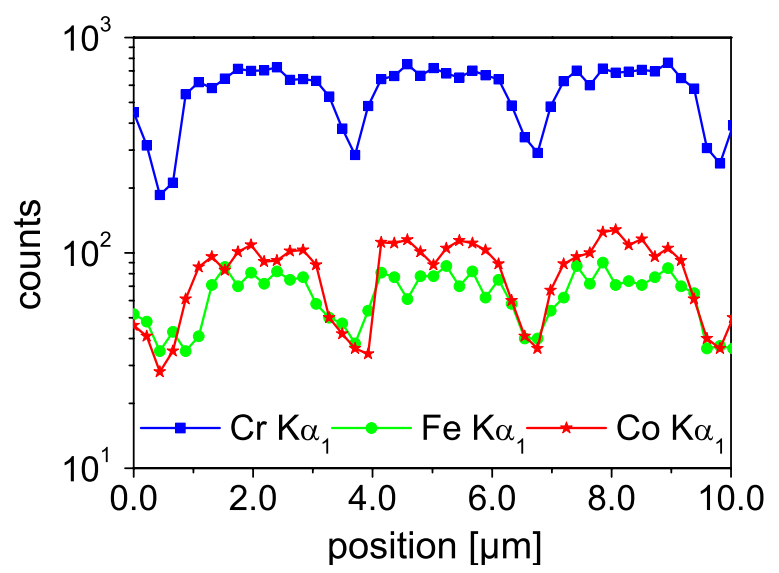

Figure 8. Integrated intensity for the different elements. The area of integration in the SEM picture with respect to the line scans shown in the panel is marked in figure 7.
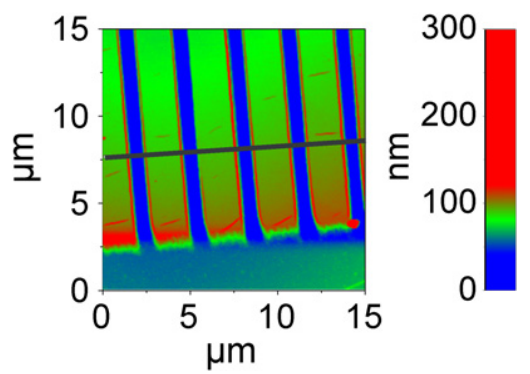

different. The Co film exhibits a very large coercivity of $180 \mathrm{mT}$, which is a result of the epitaxial film structure and its thickness [15], while the coercivity of the Fe layer is less than $1 \mathrm{mT}$. In figure 12 the azimuthal dependence of the coercivity at $50 \%$ magnetization for the continuous Co film is plotted, which exhibits the same fourfold anisotropy as the remanent magnetization. In the trilayer the coercivity is completely dominated by the Co film and will be discussed in more detail further below. Here the coercive field is taken at $20 \%$ of the saturation magnetization, because of the step in the hysteresis curve, and the coercive field values for the ascending and descending branches are averaged.

Figure 13 presents magnetic hysteresis loops for $\mathrm{Fe}$ and Co single films as well as for a $\mathrm{Co} / \mathrm{Cr} / \mathrm{Fe}$ trilayer before and after patterning. Both the longitudinal $(x)$ (along the field) and the vertical $(y)$ (perpendicular to the field) components of the magnetization reversal in the sample plane are shown in each case. In the left column the hysteresis loops of the unpatterned films with the field applied along one of the easy axes are presented. Both of the single films show only a weak $y$-component of the magnetization at the coercive fields, indicating that the reversal process is dominated by nucleation of domains and domain wall motion, with some additional rotation of moments. We note that the continuous Co film has a maximum in the $y$-component at field values, which are slightly smaller than the coercive field $H_{\mathrm{c}}$ derived from the $x$-component. This is due to the coherent rotation of the magnetic domains which starts for fields $H<H_{\mathrm{c}}$.

The trilayer hysteresis loop is typical for a spin valve structure. At the first coercive field $H_{\mathrm{Fe}}$ the Fe moments reverse. The Co moments reverse at the second coercive field $H_{\mathrm{Co}}$. In the trilayer the $y$-component of the magnetization is completely suppressed, indicating domain processes during the reversal without any rotation of moments. From this we infer the presence of a weak IEC coupling between the Fe and the Co layers mediated by the intervening $\mathrm{Cr}$ layer.

After patterning the remagnetization process is changed considerably in the single films but not very much in the trilayer as can be seen by comparing the middle and right columns with the first column of figure 13. In the middle column measurements are reproduced with the field applied perpendicular to the stripes and the right column measurements are shown with the field applied parallel to the stripes.

We first discuss the patterned Fe film. In the patterned $\mathrm{Fe}$ film only a weak $y$-component is observed when measuring with the field applied parallel to the stripes. This indicates

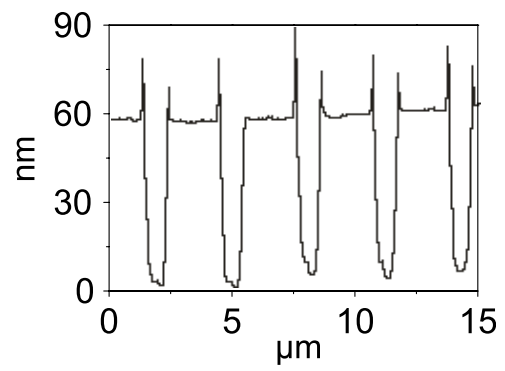

Figure 9. AFM measurement of a representative sample presenting a 3D plot of the stripes pattern (left) and a line scan across the stripes (right). (Colour online.) 


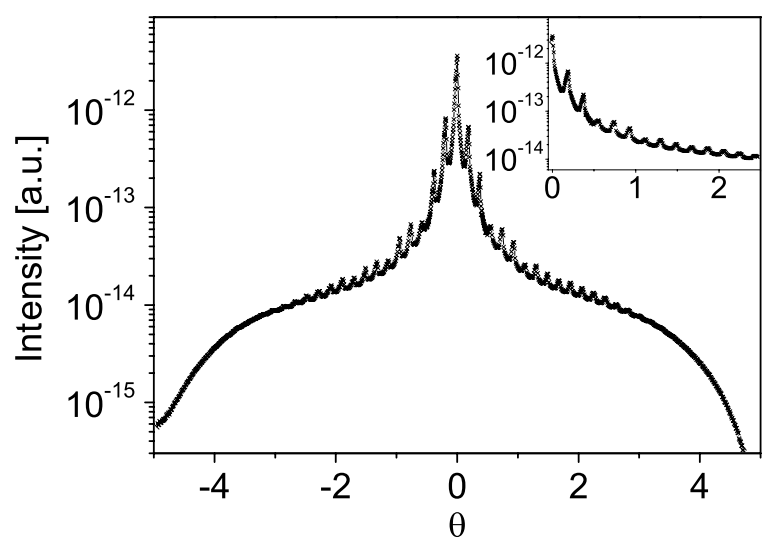

Figure 10. Rocking curves across the reflectivity of Fe stripes taken at the $L_{2}$ edge of $\mathrm{Fe}$ at $2 \theta=10^{\circ}$.

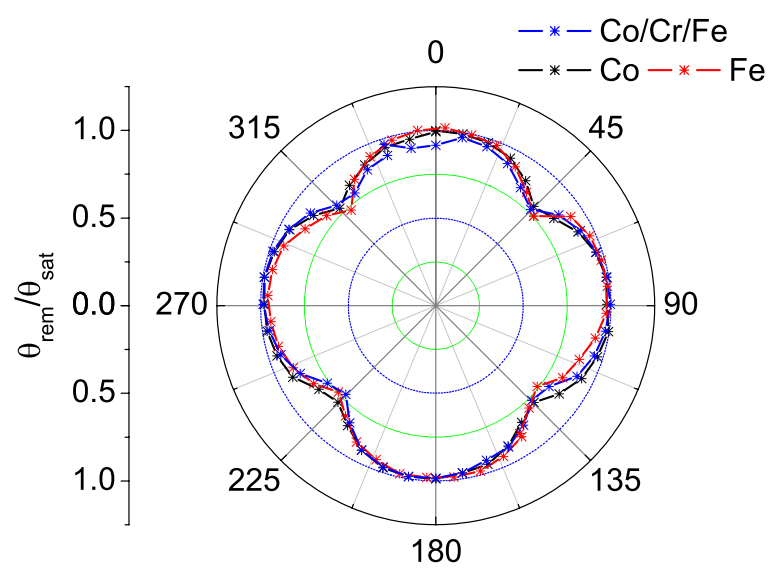

Figure 11. Azimuthal plot of the remanent magnetization as a function of sample orientation for the continuous $\mathrm{Fe}, \mathrm{Co}$ and $\mathrm{Co} / \mathrm{Cr} / \mathrm{Fe}$ films and trilayers, respectively. (Colour online.)

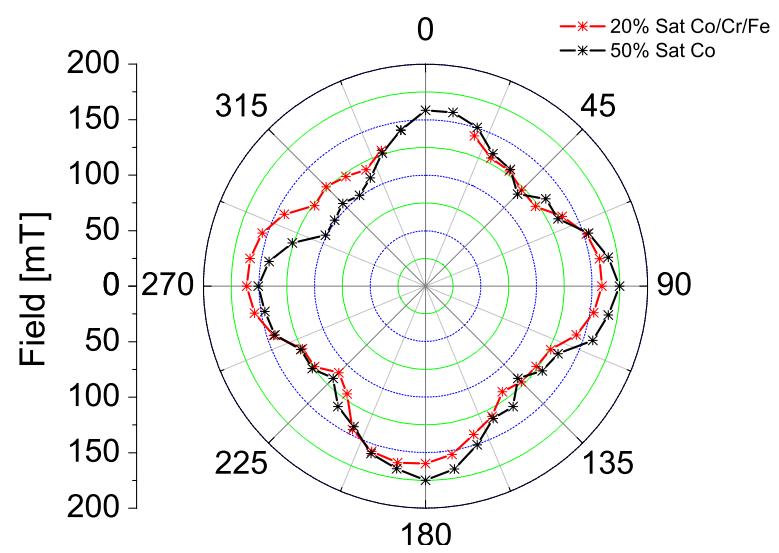

Figure 12. Azimuthal dependence of the $\mathrm{Co}$ and $\mathrm{Co} / \mathrm{Cr} / \mathrm{Fe}$ coercive fields for the continuous films are plotted as a function of sample orientation. Further details are described in the main text. (Colour online.)

that domain propagation proceeds along the stripe direction which are oriented parallel to one of the easy axes. In contrast, upon magnetization perpendicular to the stripe direction a very large $y$-component is observed, which is a clear sign of a coherent rotation process. In fact, the $y$-component reaches $100 \%$ of the magnetization during reversal. Such behaviour is only expected in the case of a single domain within the stripes. All together one can conclude that patterning implements a strong anisotropy on the magnetic reversal of Fe layers, which can be explained by a reversal through large domains.

The magnetization reversal of the Co stripes is completely different from that of the $\mathrm{Fe}$ ones. Patterning affects the reversal process in both directions parallel and perpendicular to the stripes. In the perpendicular direction all previous $y$-components are suppressed. Here, the reversal is driven entirely by nucleation processes, in strong contrast to that of the Fe stripes when applying the field perpendicular to the stripes. In the Fe stripes, the reversal is completely coherent, whereas in the Co stripes it is completely incoherent. The reversal of the Co film taken with the field parallel to the stripes exhibits a small $y$-component with a slight slope up to the coercivity for ascending as well as for descending fields. This yields a small hysteresis of the $y$-component, which is quite unusual and indicates some permanent transverse magnetization component most likely due to canted moments. This may be due to the creation of ripple domains and correlations between the moments across the stripes as was recently already observed for $\mathrm{CoFe}$ stripes [8]. Rippling is a phenomenon known to occur in Co films ([16]) and leads to an alternating canting of moments within the small domains and with respect to the applied field direction. Here, due to coupling across neighbouring stripes larger areas with tilted magnetic moments may build up, similar to recent observations by polarized neutron reflectometry (PNR) on Co stripe arrays, which exhibit hyperdomains stretching over several stripes $[8,17]$. Surprisingly and in contrast to these previous studies, here a tilt of the magnetization vectors is observed in the MOKE data, whereas before this effect was only seen by PNR. This difference was explained by the different averaging procedure that MOKE and PNR take by integrating over different magnetic domains, leading to a cancellation of the transverse signal in MOKE but not in PNR. From the fact that a small transverse signal is observed in the present data even via MOKE we infer that areas with a tilted mean magnetic moment larger than the coherence area of the laser spot with a diameter of about $500 \mu \mathrm{m}$ builds up across the Co stripes. Considering, furthermore, that Co performs a bcc-hcp transition during growth, which is accompanied by a change in the anisotropy axis within individual domains, may explain the stabilization of such a tilt.

So far it has been realized that patterning of the single layers introduces a magnetization behaviour which is changed with respect to the unpatterned film and which is also completely different for $\mathrm{Fe}$ and Co. The patterning of the trilayer is therefore expected to yield interesting results. Surprisingly, comparing the MOKE measurements from the unpatterned and the patterned trilayer films yields not much of a difference. For fields parallel to the stripes the hysteresis loops are very similar to those of the unpatterned trilayer. The $y$-component is almost completely suppressed. The Fe magnetization reversal remains very sharp and proceeds most likely via domain propagation. The Co reversal is essentially 

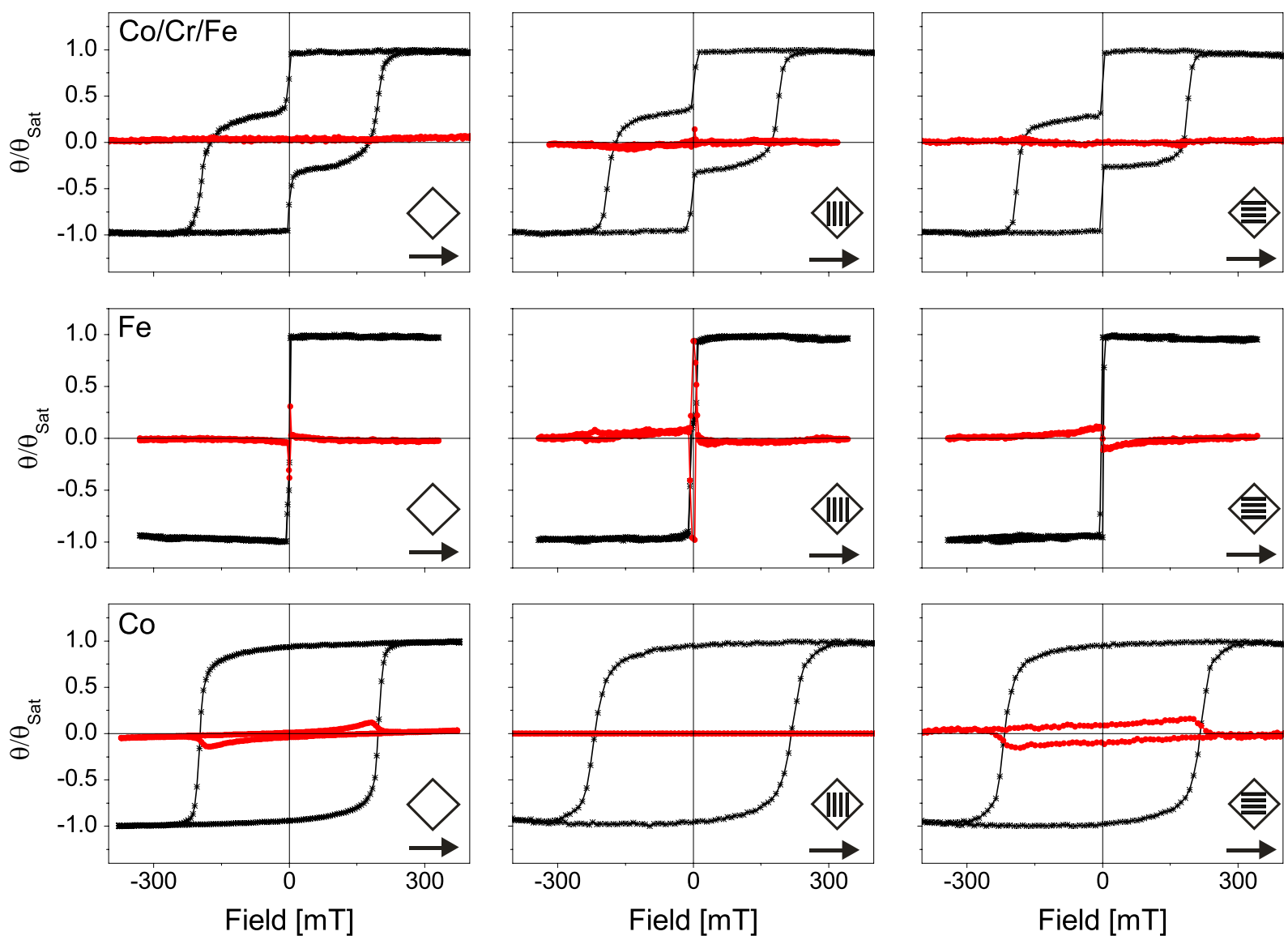

Figure 13. Vector-MOKE measurements on $\mathrm{Co} / \mathrm{Cr} / \mathrm{Fe}$ (upper row), Fe (middle row) and Co films (lower row). In the left column measurements on unpatterned samples, in the middle column measurements on the patterned samples with the magnetic field applied perpendicular to the stripes and in the right column measurements on the patterned samples with the field parallel to the stripes are shown. Crosses (dots) designate the $x(y)$-component of the magnetization, respectively. For more explanations, please refer to the main text. (Colour online.)

the same as without patterning. Even the small hysteresis in the transverse component as seen in the patterned Co film is not visible in the trilayer. Applying the field perpendicular to the stripe direction the hysteresis does not change much, aside from a small spike of the $y$-component at the coercivity of the Fe film. This spike is reminiscent of the coherent rotation of the single $\mathrm{Fe}$ stripes and shows that Fe keeps some coherent magnetization rotation. The magnetization reversal of the striped trilayer is obviously dominated by the weak interlayer coupling or by dipolar interaction between the two magnetic layers on top of each other. It is also quite surprising that any anisotropy, which is artificially introduced by the patterning, becomes strongly suppressed through this interaction and it may even be speculated that the domain nucleation in both layers is influenced by coupling effects. Furthermore, domain growth results in domains whose sizes are much smaller as compared with the domains in pure Fe films and stripes. The influence of the Fe layer on the Co magnetization may also be responsible for the suppression of any ripple domains during reversal. From this we infer that the size of the magnetic domains in the Co stripes for either parallel or perpendicular reversal is much smaller than the stripe width.

\subsection{Magnetic force microscopy}

For gaining further information on the magnetic domain configuration we performed magnetic force microscopy (MFM) studies. Figure 14 shows images of the Co stripe pattern, the Fe stripe pattern and the striped $\mathrm{Co} / \mathrm{Cr} / \mathrm{Fe}$ trilayer with $\mathrm{Co}$ at the top. The images were taken in remanence and at room temperature.

The MFM image of the Co stripes confirms very clearly the existence of small ripple domains in $\mathrm{Co}$, as already speculated from the MOKE hysteresis data. Rippling occurs over a large field range, possibly ranging from slightly negative fields towards fields somewhat larger than the coercive field for the ascending branch and similarly for the descending branch [8]. Most likely the small transverse hysteresis observed by MOKE for the field direction parallel to the stripes is connected with the rippling of the magnetization.

In contrast, the MFM image of the Fe pattern shows a completely different picture. We observe a nearly single domain state within each of the stripes with some of the stripes already reversed. Most likely the reversal proceeds via large hyperdomains as all of the left stripes have a grey MFM contrast whereas all of the right ones have a white MFM contrast with a domain boundary in between. Additionally, all Fe stripes show end domains with a darkened MFM contrast. 

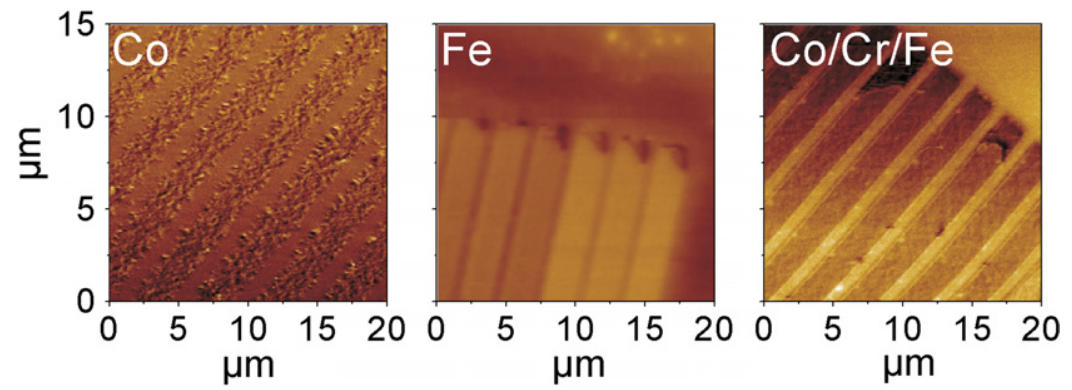

Figure 14. MFM measurements on $\mathrm{Co}, \mathrm{Fe}$ and $\mathrm{Co} / \mathrm{Cr} / \mathrm{Fe}$ stripes. (Colour online.)

The MFM image of the patterned trilayer does not show very much contrast and appears quite smooth. With some difficulties, few small domains of a similar size as seen in the Co stripes can be recognized. The low contrast is probably a result from the fact that fewer flux lines emerge due to the coupling between both magnetic layers, as was already speculated from the MOKE data. Thus, the MFM results nicely confirm the observations and conclusions already made from the MOKE data.

Finally, we would like to comment on the relation between grain size and magnetic domain size. For soft magnetic materials there is usually not a close relationship between these two quantities. The domain size observed is usually much bigger than the grain size. This is also confirmed here by the domain size in the Fe stripe array, which consists of a single domain state while the grain size (coherency volume) has a lateral extension of a few nanometres only. In the Co stripes the magnetic domain size appears to be much smaller, but still bigger than the grain size. In general, the IEC and/or dipolar fields in the samples considered here have a much bigger effect on the magnetization reversal than the grain size in these epitaxial films.

Concluding, patterning changes the reversal process of the single $\mathrm{Fe}$ and $\mathrm{Co}$ films dramatically. However, in $\mathrm{Co} / \mathrm{Cr} / \mathrm{Fe}$ trilayers exchange and/or dipolar coupling lifts the coherent rotation and the reversal becomes essentially the same for the continuous and for the patterned heterostructure. Thus in the patterned trilayers interlayer exchange interaction dominates over shape anisotropy. This is an important finding for potential integration of $\mathrm{Co} / \mathrm{Cr} / \mathrm{Fe}$ spin valves into dense arrays, such as in MRAM devices.

\section{Summary}

We have investigated the magnetization reversal of epitaxial Fe and Co films as well as of $\mathrm{Co} / \mathrm{Cr} / \mathrm{Fe}$ trilayers by vectorMOKE and MFM methods. We have compared these results with the reversal that can be observed after patterning the films and trilayers into the shape of stripes.

The results show that the magnetization reversal of epitaxial $\mathrm{Co}(11 \overline{2} 0)$ and $\mathrm{Fe}(001)$ stripe arrays is changed with respect to their unpatterned counterparts. In particular, patterning of the $\mathrm{Fe}$ film induces an additional magnetic anisotropy. Applying the field parallel to the stripe direction the domain propagation proceeds entirely along that direction. This leads to the observation of a coherent rotation when applying the field perpendicular to the stripes. For the Co film we found that the magnetization reversal is not as strongly affected by the patterning process into stripes as it is the case for $\mathrm{Fe}$. The Co reversal is characterized by a large coercive field of about $180 \mathrm{mT}$, almost $100 \%$ remanence and an incoherent reversal with a small transverse magnetization component when the field is applied along the stripes. We explain this transverse component as due to a tilt of the magnetization with respect to the applied field due to the formation of ripple domains. In contrast, the reversal of $\mathrm{Fe}$ is very sharp, with a very small coercivity of less than $1 \mathrm{mT}$ and $100 \%$ remanence.

In the case of the trilayers we find that the magnetization reversal of the continuous film and of the stripe pattern is very similar. From this we conclude that interaction between the layers via exchange or dipolar stray fields dominates over the shape anisotropy. Obviously the stripe width of $2 \mu \mathrm{m}$ is much larger than the average domain size. The large Fe domains, which are observed in the patterned Fe films, are reduced in size due to coupling to the Co stripes. Thus, in patterned $\mathrm{Co} / \mathrm{Cr} / \mathrm{Fe}$ spin valve structures proximity effects to neighbouring islands can be neglected, making them suitable for high density integration. Similar conclusions have been derived from the investigation of antiferromagnetically coupled and laterally structured $\mathrm{CoFe} / \mathrm{Ru} / \mathrm{CoFe}$ trilayers [18]. Indeed in MRAM devices the feature size is much smaller than that investigated in this work. However, we expect that by reducing the element size of the $\mathrm{Co} / \mathrm{Cr} / \mathrm{Fe}$ stacks stray field compensation still works when the individual layer thicknesses are adjusted so as to cancel out the magnetization in the remanent state.

\section{Acknowledgments}

The authors gratefully acknowledge financial support by the DFG through SFB491: Magnetic Heterostructures: Spinstructures and Spintransport, beam time allocation and support by the HASYLAB at DESY Hamburg, DELTA synchrotron at the University of Dortmund and BESSY II at the Helmoltz Zentrum Berlin. Furthermore, they would like to thank Ulrich Kunze for the use of the MFM facility, Jürgen Podschwadek for technical support as well as Kurt Westerholt, Oleg Petracic and Arndt Remhof for fruitful discussions.

\section{References}

[1] Binasch G, Grünberg P, Saurenbach F and Zinn W 1989 Phys. Rev. B 394828 
[2] Baibich M N, Broto J M, Fert A, Nguyen Van Dau F, Petroff F, Eitenne P, Creuzet G, Friedrich A and Chazelas J 1988 Phys. Rev. Lett. 612472

[3] Yamamoto H, Okuyana T, Dohnomae H and Shinjo T 1991 J. Magn. Magn. Mater. 99243

[4] Dieny B, Speriosu V S, Parin S S P, Gurney B A, Wilhoit D R and Mauri D 1991 Phys. Rev. B 431297

[5] Kimura T, Hamrle J and Otani Y 2005 Phys. Rev. B 72014461

[6] Theis-Bröhl K, Scheidt R, Zeidler Th, Schreiber F, Zabel H, Mathieu Th, Mathieu Ch and Hillebrands B 1996 Phys. Rev. B 5311613

[7] Buchmeier M, Schreiber R, Bürgler D E and Grünberg P, 2003 Europhys. Lett. 63874

[8] Theis-Bröhl K, Toperverg B P, Leiner V, Westphalen A, Zabel H, McCord J, Rott K and Brückl H 2005 Phys. Rev. B 71020403

[9] Schad R, Potter C D, Beliën P, Verbanck G, Moshchalkov V V and Bruynseraede Y 1994 Appl. Phys. Lett. 643500
[10] Donner W, Metoki N, Abromeit A and Zabel H 1993 Phys. Rev. B 4814745

[11] Metoki N, Donner W and Zabel H 1994 Phys. Rev. B 4917351

[12] Schmitte T, Theis-Bröhl K, Leiner V, Zabel H, Kirsch S and Carl A 2002 J. Phys.: Condens. Matter 147525

[13] Daboo C, Hicken R J, Gu E, Gester M, Gray S J, Eley D E P, Ahmad E, Bland J A C, Ploessl R and Chapman J N 1995 Phys. Rev. B 5115964

[14] Goryunov Yu V, Khusainov M G, Garifullin I A, Schreiber F, Pelzl J, Zeidler Th, Bröhl K, Metoki N and Zabel H 1994 J. Magn. Magn. Mater. 138216

[15] Donner W, Zeidler T, Schreiber F, Metoki N and Zabel H 1994 J. Appl. Phys. $\mathbf{7 5} 6421$

[16] Radu F, Leiner V, Westerholt K, Zabel H, McCord J, Vorobiev A, Major J, Jullien D, Humblot $\mathrm{H}$ and Tasset $\mathrm{F}$ 2005 J. Phys.: Condens. Matter 171711

[17] Theis-Bröhl K et al 2008 New J. Phys. 10093021

[18] Tezuka N, Koike N, Inomata K and Sugimoto S 2003 Appl. Phys. Lett. 82604 